\title{
Native T1 and T2 mapping by cardiovascular magnetic resonance imaging in pressure overloaded left and right heart diseases
}

\author{
Jing Wang ${ }^{1,2 \#}$, Hang Zhao ${ }^{1,3 \#}$, Yue Wang ${ }^{1,4}$, Howard C. Herrmann ${ }^{1}$, Walter R. T. Witschey ${ }^{5}$, Yuchi Han ${ }^{1}$ \\ ${ }^{1}$ Cardiovascular Division, Department of Medicine, Perelman School of Medicine, University of Pennsylvania, Philadelphia, PA, USA; ${ }^{2}$ Department \\ of Cardiology, PLA General Hospital, Beijing 100853, China; ${ }^{3}$ Department of Cardiology, Shanghai Renji Hospital, Shanghai Jiaotong University \\ School of Medicine, Shanghai 200127, China; ${ }^{4}$ Department of Cardiology, Shanghai Ninth People's Hospital, Shanghai Jiaotong University School \\ of Medicine, Shanghai 200001, China; ${ }^{5}$ Department of Radiology, Perelman School of Medicine of the University of Pennsylvania, Philadelphia, PA, \\ USA \\ Contributions: (I) Conception and design: Y Han; (II) Administrative support: Y Han; (III) Provision of study materials or patients: HC Herrmann, \\ WR Witschey, Y Han; (IV) Collection and assembly of data: J Wang, H Zhao, Y Wang, Y Han; (V) Data analysis and interpretation: All authors; (VI) \\ Manuscript writing: All authors; (VII) Final approval of manuscript: All authors. \\ \#These authors contributed equally to this work. \\ Correspondence to: Yuchi Han, MD, MMSc. Assistant Professor of Medicine, Cardiovascular Division, Perelman School of Medicine, University of \\ Pennsylvania, 11-135 South Pavilion, 3400 Civic Center Blvd, Philadelphia, PA 19104, USA. Email: yuchi.han@uphs.upenn.edu.
}

Background: Pulmonary arterial hypertension (PAH) and severe aortic valve stenosis (AS) are diseases characterized by increased afterload of the right and left heart, respectively. Our study aims to investigate the differences of myocardial tissue characteristics in the pressure overloaded left and right hearts, especially in the shared interventricular septum, as detected by native $\mathrm{T} 1$ and $\mathrm{T} 2$ relaxation times.

Methods: Eighteen patients with $\mathrm{PAH}$ and 19 patients with severe AS in addition to 5 healthy volunteers underwent 1.5-T CMR examination with native T1 and T2 mapping. Mean T1 and T2 value were measured at the right ventricular (RV) free wall, superior RV insertion, inferior RV insertion, interventricular septum and left ventricular (LV) lateral wall.

Results: Compared with controls and AS group, T1 was significantly elevated in the RV insertion in PAH group $(\mathrm{P}=0.015)$, while no statistically significant differences were seen in other segments among the three groups. There was an increase of $\mathrm{T} 2$ in the RV insertion in AS and PAH groups $(\mathrm{P}=0.01)$. Significant $\mathrm{T} 2$ elevation was also observed in the RV free wall of PAH group, and the LV lateral wall of AS group compared with the control group. RV insertion T2 was significantly correlated with RV end-diastolic volume index $(\mathrm{r}=0.608, \mathrm{P}=0.016)$ and $\mathrm{RV}$ mass index $(\mathrm{r}=0.57, \mathrm{P}=0.026)$ in the PAH group. LV lateral wall T2 and RV insertion T2 were significantly correlated with aortic valve mean gradients in the AS group $(\mathrm{r}=0.56, \mathrm{P}=0.02$; $\mathrm{r}=0.58, \mathrm{P}=0.01$, respectively).

Conclusions: In pressure overload diseases, both T1 and T2 values increase in the myocardium. The alterations seen in the RV insertion sites in the septum was more pronounced with RV pressure overload. $\mathrm{T} 2$ values also correlated with structural and functional remodeling in both diseases. Combining T1 and T2 mapping may help to better characterize the alternation of myocardial composition in pressure overloaded heart diseases.

Keywords: T1 mapping; T2 mapping; pressure overload; pulmonary hypertension; aortic stenosis

Submitted Jan 13, 2018. Accepted for publication Apr 19, 2018.

doi: $10.21037 /$ jtd.2018.04.141

View this article at: http://dx.doi.org/10.21037/jtd.2018.04.141 


\section{Introduction}

Native T1 and T2 myocardial mapping techniques offer quantitative assessments of the myocardium composition by measuring $\mathrm{T} 1$ and $\mathrm{T} 2$ relaxation times, which are fundamental tissue properties of the myocardium that change in the diseased states (1). Pulmonary arterial hypertension $(\mathrm{PAH})$ is a pulmonary vasculopathy defined by elevated resting mean pulmonary artery pressure (mPAP) $>25 \mathrm{mmHg}$ in the setting of a normal pulmonary capillary wedge pressure (PCWP) $<15 \mathrm{mmHg}$ (2). PAH is characterized by excess proliferation, apoptosis resistance, inflammation, fibrosis and vasoconstriction in the pulmonary vasculature and with resulting pressure overload on the right side of the heart (3). Severe calcific aortic valve stenosis (AS) in the elderly may cause cardiomyocyte hypertrophy, apoptosis, and myofibroblast activation, which result in myocardial fibrosis, due to the excessive pressure overload on the left side of the heart (4). For patients with PAH, the right heart pressure overload is dominant. For patients with AS, the left heart pressure overload is dominant. A number of studies have examined native tissue properties in both diseases (5-9). How do these two pressure overload situations compare and differ in terms of the tissue characteristics of the left ventricle, right ventricle, and the septum which is shared by both ventricles had not been previously studied. Our study aimed to investigate the differences in the pressure overloaded left heart versus right heart as detected by native $\mathrm{T} 1$ and $\mathrm{T} 2$ relaxation times.

\section{Methods}

\section{Patient selection}

We prospectively recruited 22 patients with $\mathrm{PAH}$ and 21 patients with severe AS in addition to 5 healthy volunteers who all underwent CMR examination with native T1 and T2 mapping. The healthy controls were volunteers without any history of hypertension, hyperlipidemia, diabetes, or any other cardiovascular diseases and are on no medications. For the PAH patients, they were diagnosed by right heart catheterization, meeting diagnostic criteria for $\mathrm{PAH}$, were on stable $\mathrm{PAH}$ specific medications for at least 3 months and had no medication dosage change for at least 28 days. Patients with severe aortic stenosis were under evaluation for transcatheter aortic valve replacement. Physical examination (for weight, height), left and right heart catheterization, and echocardiography (for aortic valve mean gradients and aortic area) was conducted at the time of CMR or at a clinic visit before CMR. The study was approved by our Institutional Review Board and all patients gave written informed consent.

\section{CMR protocol}

All patients underwent a CMR examination using a 1.5-T MRI scanner (Avanto, Siemens, Germany) equipped with 16-channel anterior and posterior receiver arrays. Cine imaging was performed using steady-state free precession (SSFP) to assess left ventricular (LV) and right ventricular (RV) dimensions, function, and mass. Retrospectively ECGgated cine MRI was performed with the following sequence parameters: $\mathrm{TE} / \mathrm{TR}=1.07 / 2.2 \mathrm{~ms}$; slice thickness $=8 \mathrm{~mm}$; slice gap $=2 \mathrm{~mm}$; bandwidth $=930 \mathrm{~Hz} /$ pixel; flip angle $=70^{\circ}$; field of-view $=380 \times 310 \mathrm{~mm}^{2}$; spatial resolution $=1.97 \times 1.97 \mathrm{~mm}^{2}$; parallel imaging factor $=2$.

Native T1 maps were acquired at mid-ventricle using a modified Look-Locker inversion recovery (MOLLI) sequence (10) in diastole using the following sequence parameters: $\mathrm{TE} / \mathrm{TR}=1.17 / 2.4 \mathrm{~ms}$; minimum $\mathrm{TI}=100 \mathrm{~ms}$; TI increment $=80 \mathrm{~ms}$; flip angle $=35^{\circ}$, field of-view $=240 \times 330 \mathrm{~mm}^{2}$; spatial resolution $=1.71 \times 1.67 \mathrm{~mm}^{2}$ (interpolated to $1.28 \times 1.28 \mathrm{~mm}^{2}$ ); slice thickness $=8 \mathrm{~mm}$; bandwidth $=1,085 \mathrm{~Hz} /$ pixel; parallel imaging factor $=2$.

T2 maps were acquired at mid-ventricle using a T2prepared SSFP sequence with the following sequence parameters at end-systole: $\mathrm{T} 2$-preparation durations $=0,24$, and $55 \mathrm{~ms}$; TE $/ \mathrm{TR}=1.37 / 2.64 \mathrm{~ms}$; flip angle $=35^{\circ}$; field-ofview $=270 \times 360 \mathrm{~mm}^{2}$; bandwidth $=1,185 \mathrm{~Hz} /$ pixel, matrix $=$ $192 \times 120$ pixels, spatial resolution $=1.88 \times 2.25 \mathrm{~mm}$ (interpolated to $0.93 \times 0.93 \mathrm{~mm}^{2}$ ), slice thickness $=8 \mathrm{~mm}$; parallel imaging factor $=2$.

\section{Image analysis}

$\mathrm{LV}$ and $\mathrm{RV}$ mass, volume and ejection fraction were obtained by manually tracing the endocardial (excluding papillary muscles) and epicardial borders according to SCMR recommendations (11) using Qmass software (Medis, Netherlands). For T1 and T2 analyses, regions-of-interest were manually drawn at the RV free wall, superior RV insertion, inferior $\mathrm{RV}$ insertion, interventricular septum and LV lateral wall (Aquarius iNtuition, TeraRecon, Foster City, USA). RV insertion values were reported as an average of superior and inferior RV insertions. 
Table 1 Baseline demographics and cardiac function

\begin{tabular}{|c|c|c|c|c|}
\hline Characteristics & $\mathrm{PAH}(\mathrm{n}=18)$ & AS $(n=19)$ & Control $(n=5)$ & $\mathrm{P}$ \\
\hline Sex (male) & $8(44.4 \%)$ & $11(57.9 \%)$ & $3(60 \%)$ & 0.60 \\
\hline $\mathrm{BSA}\left(\mathrm{m}^{2}\right)$ & $2.0 \pm 0.3$ & $1.9 \pm 0.2$ & $1.8 \pm 0.3$ & 0.48 \\
\hline \multicolumn{5}{|l|}{ Cardiac function } \\
\hline LVEF (\%) & $55.8 \pm 6.3$ & $43.9 \pm 15.3$ & $60 \pm 2.5$ & 0.01 \\
\hline LV Mass Index $\left(\mathrm{g} / \mathrm{m}^{2}\right)$ & $43.7 \pm 13.4$ & $79.7 \pm 20.7$ & $42.5 \pm 5.2$ & $<0.001$ \\
\hline RVEDVi $\left(\mathrm{mL} / \mathrm{m}^{2}\right)$ & $121.4 \pm 64.5$ & $75.9 \pm 25$ & $90.4 \pm 12.5$ & 0.002 \\
\hline RVEF (\%) & $37.8 \pm 10.6$ & $54.1 \pm 13.9$ & $55.1 \pm 3.2$ & 0.001 \\
\hline Aortic area $\left(\mathrm{m}^{2}\right)$ & - & $0.6 \pm 0.1$ & - & - \\
\hline Mean PAP (mmHg) & $43.53 \pm 12.72$ & $27.31 \pm 9.75$ & - & $<0.001$ \\
\hline
\end{tabular}

AS, aortic stenosis; BSA, body surface area; EF, ejection fraction; LV, left ventricular; LVEDVi, left ventricular end-diastolic volume index; $\mathrm{PAH}$, pulmonary arterial hypertension; PAP, pulmonary arterial pressure; RV, right ventricular; RVEDVi, right ventricular end-diastolic volume index.

\section{Intra- and inter-observer reproducibility}

Ten studies were randomly selected for reproducibility analysis. For inter-observer reproducibility, two observers independently analyzed the T1 and T2 data at all locations for the ten studies. For inter-observer reproducibility, one observer repeated the measurements on the same studies 1 week later. Intraclass correlation coefficient was used to assess reproducibility.

\section{Statistical analysis}

All continuous variables including cardiac volumes and functional parameters, pressure gradients, and T1 and T2 measurements are presented as mean $\pm \mathrm{SD}$. Categorical variables are expressed as percentages. Baseline characteristics and T1 and T2 measurements among three groups were compared with Kruskal-Wallis tests. The difference between multiple groups were assessed by Mann-Whitney U test. Categorical variables among groups were compared with Chi-square test. A P value of $<0.05$ was considered significant. Correlations were assessed by Spearman's correlation coefficient. All statistical analysis was carried out using SPSS 17.0 (SPSS Inc., Chicago, USA). Linear regression was used to explore the effect of age on mapping parameters.

\section{Results}

\section{Patient characteristics}

A total of 42 subjects were included in the analysis based on adequate image quality. This included 18 subjects with PAH, 19 with severe AS, and 5 healthy controls. Baseline characteristics and cardiac function were listed on Table 1 . The average age of the three groups was $61 \pm 12,85 \pm 5$, and $37 \pm 13$ years, respectively $(\mathrm{P}<0.001)$. However, linear regression showed that age had no effect on $\mathrm{T} 1$ and $\mathrm{T} 2$ measurements $(\mathrm{P}=0.16$ and 0.79 , respectively) in this cohort of patients. Compared with controls, $\mathrm{PAH}$ group had lower RVEF and increased RV mass index $(\mathrm{P}<0.001$, respectively). The AS group had a lower LVEF and increased $\mathrm{LV}$ mass and $\mathrm{RV}$ mass indices $(\mathrm{P}=0.015,0.001$ and 0.005 , respectively). The mean pulmonary systolic pressure of the AS group was slightly elevated from normal level $(27.3 \pm 9.8 \mathrm{mmHg})$, but was lower compared with the $\mathrm{PAH}$ group $(43.5 \pm 12.7 \mathrm{mmHg}, \mathrm{P}<0.001)$.

\section{Comparison of T1 and T2 measurements among groups}

Native T1 and T2 values are listed in Table 2 and an example of each group is shown in Figure 1. T1 was significantly 
Table 2 Native T1 and T2 values at different ventricular locations

\begin{tabular}{|c|c|c|c|c|}
\hline Groups & Septum & LV lateral & Insertion & RV free wall \\
\hline \multicolumn{5}{|l|}{$\mathrm{T} 1$ (ms) } \\
\hline PAH & $1056.2 \pm 58.6$ & $997.3 \pm 65.7$ & $1129.7 \pm 61.7^{\star \#}$ & $1035.93 \pm 78.59$ \\
\hline AS & $1052.3 \pm 62.1$ & $1018.8 \pm 55.5$ & $1062.35 \pm 132.8$ & $983.92 \pm 78.31$ \\
\hline Control & $1011.6 \pm 21.0$ & $984.0 \pm 43.5$ & $1036.1 \pm 63.8$ & $947.94 \pm 59.91$ \\
\hline PAH & $46.4 \pm 4.4$ & $47.1 \pm 4.4$ & $53 \pm 4.6^{\star \#}$ & $47.6 \pm 3.5^{\star \#}$ \\
\hline AS & $46.0 \pm 2.9$ & $47.3 \pm 3.6^{\Delta}$ & $48.4 \pm 1.9^{\Delta}$ & $44.1 \pm 4.6$ \\
\hline Control & $44.6 \pm 2.8$ & $42.8 \pm 2.4$ & $42.5 \pm 3.3$ & $41.0 \pm 2.9$ \\
\hline
\end{tabular}

*, $\mathrm{P}<0.05$ PAH versus Control group; ", $\mathrm{P}<0.05 \mathrm{PAH}$ versus AS group; $\Delta, \mathrm{P}<0.05$ AS versus Control group. AS, aortic stenosis; LV, left ventricular; $\mathrm{PAH}$, pulmonary arterial hypertension; $\mathrm{RV}$, right ventricular.

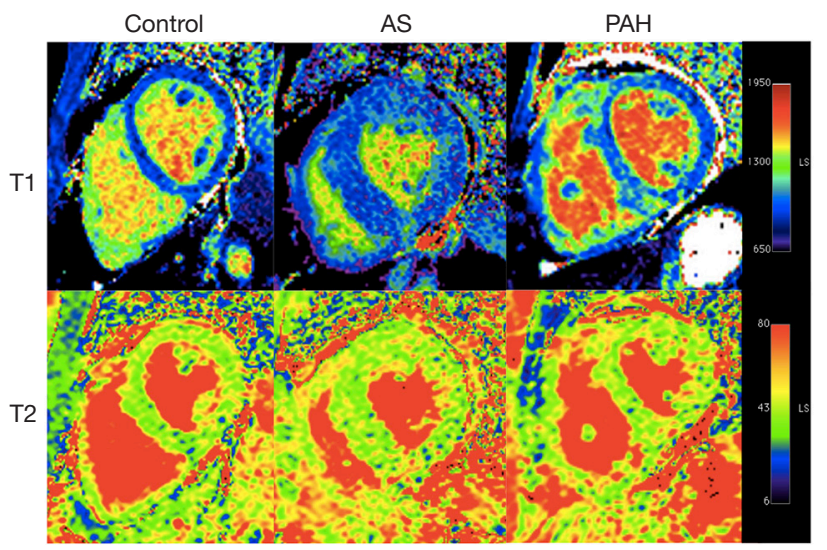

Figure 1 T1 and T2 mapping images in control, aortic stenosis and pulmonary arterial hypertension patients. PAH, pulmonary arterial hypertension; AS, aortic valve stenosis.

elevated in the RV insertion in PAH group as compared with controls and the AS group ( $\mathrm{P}=0.015$ and 0.024 , respectively). No statistically significant differences in $\mathrm{T} 1$ were seen in other segments among three groups. For T2 mapping, there was an increase of $\mathrm{T} 2$ in the RV insertion in the AS and PAH groups, with the $\mathrm{PAH}$ group having the highest $\mathrm{T} 2$ values. Compared with controls, significant $\mathrm{T} 2$ elevation was also observed in the RV free wall of the PAH group, and the LV lateral wall of the AS group (Figure 2).

\section{Correlations between tissue characteristics and cardiac functional parameters}

RV insertion T2 was significantly correlated with RVEDVi $(\mathrm{r}=0.608, \mathrm{P}=0.016)$ and $\mathrm{RV}$ mass index $(\mathrm{r}=0.57, \mathrm{P}=0.026)$ in the PAH group (Figure $3 A, B)$. LV lateral wall and $\mathrm{RV}$ insertion T2 was significantly correlated with aortic valve mean gradients in the AS group $(\mathrm{r}=0.56, \mathrm{P}=0.02$; $\mathrm{r}=0.58, \mathrm{P}=0.01$, respectively) (Figure $3 C, D)$. There were no significant correlations between $\mathrm{T} 1$ values and cardiac functional parameters.

\section{Reproducibility}

The intra- and inter-observer reproducibility results using intraclass correlation coefficients are shown in Table 3.

\section{Discussion}

We investigated the effect of increased $\mathrm{LV}$ and $\mathrm{RV}$ afterload on tissue properties assessed by CMR using native T1 and T2 mapping and we have found increased native T2 value in LV lateral wall in the AS group and in RV free wall in the PAH group. For the interventricular septum, there is no difference in T1 T2 measurements in the mid-septum among the three groups. However, the elevation of both $\mathrm{T} 1$ and $\mathrm{T} 2$ at RV insertion (average of superior and inferior insertions) was more prominent in the RV pressure overload compared with $L V$ pressure overload.

Elevated native T1 has been associated with pathologies that increase the extracellular compartment volume, such as diffuse fibrosis $(6,12)$ and amyloidosis (13). Our finding of elevated $\mathrm{T} 1$ values in the $\mathrm{RV}$ insertion in PAH patients is consistent with previous $\mathrm{T} 1$ and late gadolinium enhancement (LGE) findings in PAH $(14,15)$. On the other hand, T2 


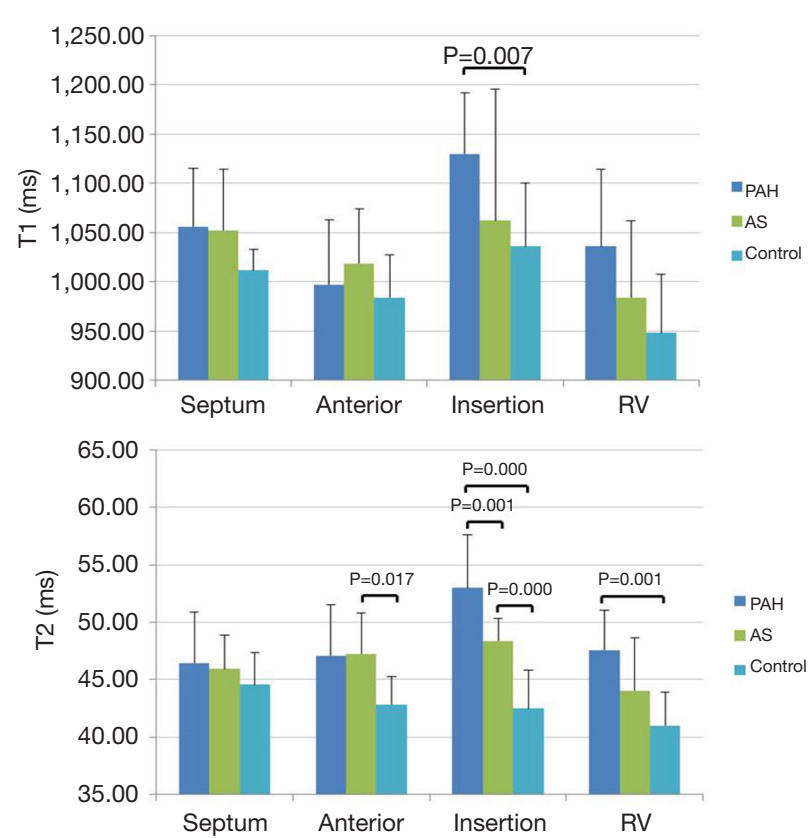

Figure 2 Comparisons of $\mathrm{T} 1$ and $\mathrm{T} 2$ among control, aortic stenosis, and pulmonary arterial hypertension groups in different parts of the ventricular walls. PAH, pulmonary arterial hypertension; AS, aortic valve stenosis; RV, right ventricular; LV, left ventricular.
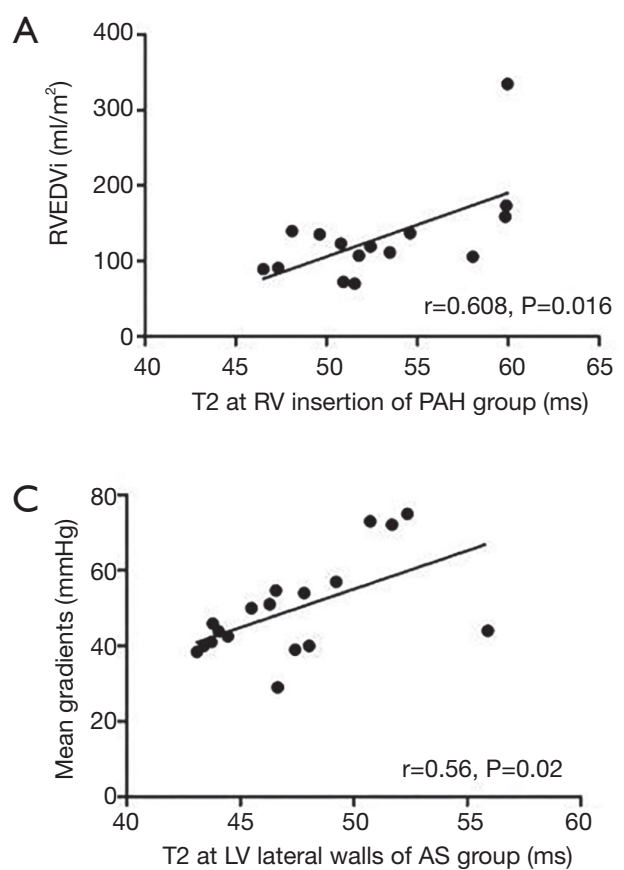

Table 3 Reproducibility using intraclass correlation coefficients of native $\mathrm{T} 1$ and $\mathrm{T} 2$

\begin{tabular}{lcc}
\hline Locations & Intra-observer & Inter-observer \\
\hline T1 septum & 0.963 & 0.953 \\
T1 LV lateral & 0.906 & 0.888 \\
T1 superior insertion & 0.991 & 0.958 \\
T1 inferior insertion & 0.981 & 0.864 \\
T1 RV free wall & 0.988 & 0.802 \\
T2 septum & 0.931 & 0.959 \\
T2 LV Lateral & 0.944 & 0.967 \\
T2 superior insertion & 0.980 & 0.975 \\
T2 inferior insertion & 0.889 & 0.901 \\
T2 RV free wall & 0.963 & 0.851 \\
\hline
\end{tabular}

$\mathrm{LV}$, left ventricular; $\mathrm{RV}$, right ventricular.

B

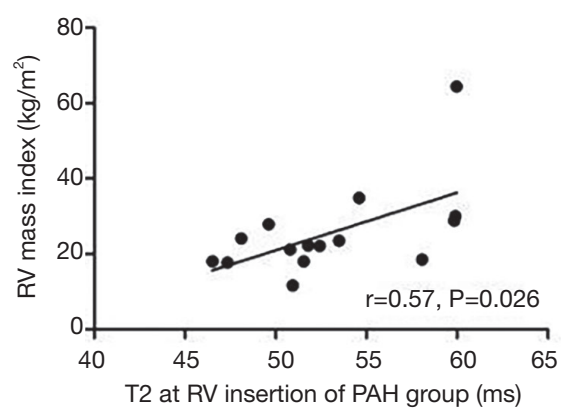

$\mathrm{D}$

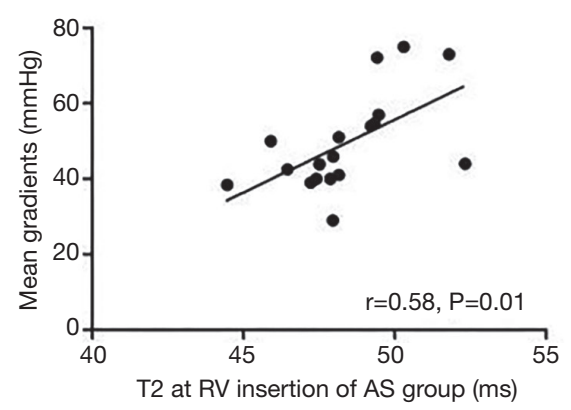

Figure 3 Correlations between T2 and RV volume (A), and mass (B) in PAH patients and correlations between aortic mean gradient and LV lateral wall T2 (C) and RV insertion T2 (D) in aortic stenosis patients. PAH, pulmonary arterial hypertension; AS, aortic valve stenosis; RV, right ventricular; $\mathrm{LV}$, left ventricular. 
relaxation time represents the time constant governing the exponential decay of transverse magnetization. The fractional increase in $\mathrm{T} 2$ is substantially larger than the fractional increase in T1 when water content is increased, making it a good technique for detecting myocardial edema (16). Its clinical significance has been validated in acute or inflammation-related diseases such as myocardial infarction, myocarditis, Takotsubo cardiomyopathy, and heart transplant rejection (16). However, the mechanisms determining the changes of $\mathrm{T} 1$ and $\mathrm{T} 2$ are complex and cross correlations are present in many situations. For example, $\mathrm{T} 1$ also increase in acute edema and inflammation (17-19), and the elevation of T2 could also be observed in fibrosis (20). In-vivo study also reported the elevation of T2 in fat (21). Our study was the first to combine T1 and T2 mapping techniques in comparing AS and $\mathrm{PAH}$ patients.

The pathological change of AS is characterized by diffuse fibrosis due to increased LV afterload, which progresses to irreversible focal replacement fibrosis, leading to $\mathrm{LV}$ decompensation (7). Elevation of native $\mathrm{T} 1$ in $\mathrm{LV}$, along with increased extracellular volume (ECV) has been reported $(6,8,22)$. Our study did not detect statistical difference of $\mathrm{T} 1$ values in the $\mathrm{LV}$ lateral wall between AS group and controls may result from our relatively small sample size, and the high overlapping T1 values, which was also noted in previous studies (23). However, T2 value increased in the LV lateral wall and RV insertion of AS group. Although the underlying mechanism is not clear, the correlation between T2 measurements and mean aortic gradients suggests that $\mathrm{T} 2$ mapping might be an interesting marker that is not limited to edema.

Compared with controls, T2 in the RV free wall of PAH group was elevated, while T1 was not. The process of RV remodeling with pressure overload might be different from the LV. The fibrosis might be much less extensive, which helps to explain why the majority of patients recover their RV function after lung transplantation, even with severely reduced RVEF (24). This also helps to explain why T1 change was not significant in RV free wall in PAH patients. On the other hand, the elevation of T2 in RV free wall in $\mathrm{PAH}$ may imply the underlying pathological changes other than fibrosis with increased fluid (blood) content in the RV, or increased fat content in myocardium due to metabolic change, or edema caused by inflammation process that usually involved in the setting of RV failure (25).

Compared with LV and RV free walls, the interventricular septum, consisted of the mid-septum and RV insertions, is shared by two ventricular chambers. Previous studies suggested that in patients with chronic thromboembolic pulmonary hypertension, the change of septum followed a RV pressure overloading pattern while the LV free wall mass index decreased (26). Similarly, we found no difference in mid-septal T1 and T2 parameters between PAH and AS groups. However, the RV insertions were more affected in right heart pressure overload compared with left heart pressure overload. The points of RV insertion are considered to bear the most significant mechanical stress when RV pressure is elevated (14), with pathological evidence of regional fibrosis proliferation and increased fat content (27). Our study showed significant elevation of both T1 and $\mathrm{T} 2$ value in $\mathrm{RV}$ insertions and correlation between RV insertion T2 with RV remodeling parameters in the PAH group, which was similar with previous T1 studies $(5,14,15)$. Compared with triangularly shaped RV, the LV geometry is more symmetrical and the increased pressure is exerted on all ventricular segments, the impact might be more diffuse and less severe in contrast with the focal insertion points in $\mathrm{RV}$ pressure overloaded hearts. That may explain the less prominent elevation of insertion $\mathrm{T} 1$ and $\mathrm{T} 2$ values in the AS group compared with the PAH group. However, the increase of RV insertion T2 may reveal the impact of the mildly elevated RV pressure in the AS group since the elevation of $\mathrm{T} 2$ is correlated with the increase of mPAP and $\mathrm{RV}$ mass index in AS patients before significant increase of RVEDVi and decrease of RVEF become apparent. This suggests that native T2 may have potential to detect early myocardial impairments in RV remodeling in the setting of predominant $\mathrm{LV}$ disease.

The major limitation of the study is the small sample size from a single center. The results are preliminary but suggest the potential of combining $\mathrm{T} 1$ and $\mathrm{T} 2$ mapping in early detection, evaluation, and management of both left and right heart pressure-overload diseases. Future larger and multi-centered studies are necessary to confirm the findings, explore the pathological mechanisms underlying the changes of these CMR parameters, and to understand the prognostic significance of our findings.

\section{Conclusions}

In pressure overload diseases, both native $\mathrm{T} 1$ and $\mathrm{T} 2$ values increase in the myocardium. The effects on $\mathrm{T} 1$ and $\mathrm{T} 2$ are more dramatic in $\mathrm{RV}$ pressure overload as compared to $\mathrm{LV}$ pressure overload in the RV insertions of the septum. Combining T1 and T2 mapping may help us better 
characterize the alternation of myocardial composition in pressure overloaded heart diseases.

\section{Acknowledgements}

This study was supported by a research grant from Cardiovascular Medical Research and Education Fund, Philadelphia, PA to Y Han.

\section{Footnote}

Conflicts of Interest: The authors have no conflicts of interest to declare.

Ethical Statement: The study was approved by our Institutional Review Board (No. 819533, 817786, and 815442) and all patients gave written informed consent.

\section{References}

1. Puntmann VO, Voigt T, Chen Z, et al. Native T1 mapping in differentiation of normal myocardium from diffuse disease in hypertrophic and dilated cardiomyopathy. JACC Cardiovasc Imaging 2013;6:475-84.

2. Hoeper MM, Bogaard HJ, Condliffe R, et al. Definitions and diagnosis of pulmonary hypertension. J Am Coll Cardiol 2013;62:D42-50.

3. Ryan JJ, Huston J, Kutty S, et al. Right ventricular adaptation and failure in pulmonary arterial hypertension. Can J Cardiol 2015;31:391-406.

4. Milano AD, Faggian G, Dodonov M, et al. Prognostic value of myocardial fibrosis in patients with severe aortic valve stenosis. J Thorac Cardiovasc Surg 2012;144:830-7.

5. Mehta BB, Auger DA, Gonzalez JA, et al. Detection of elevated right ventricular extracellular volume in pulmonary hypertension using Accelerated and NavigatorGated Look-Locker Imaging for Cardiac T1 Estimation (ANGIE) cardiovascular magnetic resonance. J Cardiovasc Magn Reson 2015;17:110.

6. Bull S, White SK, Piechnik SK, et al. Human non-contrast $\mathrm{T} 1$ values and correlation with histology in diffuse fibrosis. Heart 2013;99:932-7.

7. Debl K, Djavidani B, Buchner S, et al. Delayed hyperenhancement in magnetic resonance imaging of left ventricular hypertrophy caused by aortic stenosis and hypertrophic cardiomyopathy: visualisation of focal fibrosis. Heart 2006;92:1447-51.

8. Chin CW, Semple S, Malley T, et al. Optimization and comparison of myocardial $\mathrm{T} 1$ techniques at $3 \mathrm{~T}$ in patients with aortic stenosis. Eur Heart J Cardiovasc Imaging 2014;15:556-65.

9. Swift AJ, Rajaram S, Capener D, et al. LGE Patterns in Pulmonary Hypertension Do Not Impact Overall Mortality. JACC: Cardiovascular Imaging 2014;7:1209-17.

10. Messroghli DR, Radjenovic A, Kozerke S, et al. Modified Look-Locker inversion recovery (MOLLI) for highresolution T1 mapping of the heart. Magn Reson Med 2004;52:141-6.

11. Schulz-Menger J, Bluemke DA, Bremerich J, et al. Standardized image interpretation and post processing in cardiovascular magnetic resonance: Society for Cardiovascular Magnetic Resonance (SCMR) Board of Trustees Task Force on Standardized Post Processing. J Cardiovasc Magn Reson 2013;15:35.

12. Kuruvilla S, Janardhanan R, Antkowiak P, et al. Increased extracellular volume and altered mechanics are associated with LVH in hypertensive heart disease, not hypertension alone. JACC Cardiovasc Imaging 2015;8:172-80.

13. Karamitsos TD, Piechnik SK, Banypersad SM, et al. Noncontrast T1 mapping for the diagnosis of cardiac amyloidosis. JACC Cardiovasc Imaging 2013;6:488-97.

14. Patel AR, Addetia K. Prediction of Prognosis in Pulmonary Hypertension Using CMR What Happens Where the Right and Left Ventricles Meet? JACC Cardiovasc Imaging 2014;7:1218-20.

15. Garcia-Alvarez A, Garcia-Lunar I, Pereda D, et al. Association of myocardial T1-mapping CMR with hemodynamics and $\mathrm{RV}$ performance in pulmonary hypertension. JACC Cardiovasc Imaging 2015;8:76-82.

16. Ferreira VM, Piechnik SK, Robson MD, et al. Myocardial tissue characterization by magnetic resonance imaging: novel applications of $\mathrm{T} 1$ and $\mathrm{T} 2$ mapping. J Thorac Imaging 2014;29:147-54.

17. Higgins CB, Herfkens R, Lipton MJ, et al. Nuclear magnetic resonance imaging of acute myocardial infarction in dogs: alterations in magnetic relaxation times. Am J Cardiol 1983;52:184-8.

18. Ugander M, Bagi PS, Oki AJ, et al. Myocardial Edema as Detected by Pre-Contrast T1 and T2 CMR Delineates Area at Risk Associated With Acute Myocardial Infarction. JACC Cardiovasc Imaging 2012;5:596-603.

19. Ferreira VM, Piechnik SK, Dall'Armellina E, et al. T1 Mapping for the Diagnosis of Acute Myocarditis Using CMR: Comparison to T2-Weighted and Late Gadolinium Enhanced Imaging. JACC Cardiovasc Imaging 2013;6:1048-58. 
20. Caudron J, Mulder P, Nicol L, et al. MR relaxometry and perfusion of the myocardium in spontaneously hypertensive rat: correlation with histopathology and effect of anti-hypertensive therapy. Eur Radiol 2013;23:1871-81.

21. Han E, Gold G, Stainsby J. In-Vivo T1 and T2 Measurements of Muskuloskeletal Tissue at 3T and 1.5T. Proc Intl Soc Mag Reson Med 2003;11:450.

22. Lee SP, Lee W, Lee JM, et al. Assessment of Diffuse Myocardial Fibrosis by Using MR Imaging in Asymptomatic Patients with Aortic Stenosis. Radiology 2015;274:359-69.

23. Everett RJ, Stirrat CG, Semple SI, et al. Assessment of myocardial fibrosis with T1 mapping MRI. Clin Radiol 2016;71:768-78.

24. Vonk-Noordegraaf A, Haddad F, Chin KM, et al. Right

Cite this article as: Wang $\mathrm{J}$, Zhao $\mathrm{H}$, Wang $\mathrm{Y}$, Herrmann HC, Witschey WR, Han Y. Native T1 and T2 mapping by cardiovascular magnetic resonance imaging in pressure overloaded left and right heart diseases. J Thorac Dis 2018;10(5):2968-2975. doi: 10.21037/jtd.2018.04.141 heart adaptation to pulmonary arterial hypertension: physiology and pathobiology. Journal of the American College of Cardiology 2013;62:D22-33.

25. von Haehling S, von Bardeleben RS, Kramm T, et al. Inflammation in right ventricular dysfunction due to thromboembolic pulmonary hypertension. Int J Cardiol 2010;144:206-11.

26. Hardziyenka M, Campian ME, Reesink HJ, et al. Right ventricular failure following chronic pressure overload is associated with reduction in left ventricular mass: evidence for atrophic remodeling. J Am Coll Cardiol 2011;57:921-8.

27. Bradlow WM, Assomull R, Kilner PJ, et al. Understanding late gadolinium enhancement in pulmonary hypertension. Circ Cardiovasc Imaging 2010;3:501-3. 